\title{
A Simplified Multifractal Model for Self-Similar Traffic Flows in High-Speed Computer Networks
}

\author{
Ginno Millán \\ Universidad San Sebastián \\ ginno.millan@uss.cl
}

\begin{abstract}
This paper proposes a multifractal model, with the aim of providing a possible explanation for the locality phenomenon that appears in the estimation of the Hurst exponent in stationary second order temporal series representing self-similar traffic flows in current high-speed computer networks. It is shown analytically that this phenomenon occurs if the network flow consists of several components with different Hurst exponents.
\end{abstract}

Keywords. Multifractals, Self-similarity, Hurst exponent $(H)$, High-speed computer networks, Traffic models.

\section{Un Modelo Multifractal Simplificado para Flujos de Tráfico Autosimilares en Redes de Computadoras de Alta Velocidad}

Resumen. Se propone un modelo multifractal, con el ánimo de proveer una posible explicación al fenómeno de localidad que aparece en la estimación del exponente de Hurst en series temporales estacionarias de segundo orden, propias de los flujos de tráfico autosimilares en las actuales redes de computadoras de alta velocidad. Analíticamente se demuestra que este fenómeno se presenta cuando los flujos se componen de diversos tipos de tráficos con diferentes exponentes de Hurst.

Palabras clave. Multifractales, autosimilitud, exponente de Hurst $(H)$, redes de computadoras de alta velocidad, modelos de tráfico.

\section{Introduction}

The properties that evidence the nature of the fractal origin of traffic flows in high-speed computer networks have been extensively studied and reported in the literature during the last twenty years, and it is generally accepted that their rescaled dynamic behavior must be carefully considered in performance analyses.
There are, therefore, numerous explanations and models that attempt to give an answer to this origin, e.g. [1-3].

On the other hand, admitting that the localities of a fractal process can only be analyzed from the standpoint of multifractal analysis, in view of their construction from multiplicative cascades that ensure an exact characterization as a result of the high frequency analysis $[9,10]$, it is accepted that the traffic flows present in current high-speed computer networks are of a multifractal nature, and this gives rise to a new flow model that attempts to explain the locality phenomenon present in the estimation of the Hurst exponent [4, $8,10]$.

From the results obtained by the use of computational simulations, it is inferred that the model contributes to the knowledge of the actual dynamics of the traffic in current high-speed computer networks, and that it can be used to simulate realistic traffic flow from real networks.

\section{On the Multifractal Traffic Flows}

In computer networks traffic flows is represented by means of a self-similar process $Y(k)$ such that

$$
Y(k)={ }_{d} a^{-H} Y(a k), \quad \forall a>0, k \geq 0,
$$

where $=_{d}$ represents the equal finite-dimensional distributions and $H \in(0,1)$ is the Hurst exponent of the self-similar stochastic process $(H-S S) Y(k)$.

An $\mathrm{H}$-SS process with stationary increments described in terms of the behavior of its aggregations is obtained by the multiplexation of $X(k)=Y(k+1)-Y(k)$ increments over nonsuperposed blocks of size $n$ according to 


$$
X^{(n)}(k)=\sum_{j=0}^{n-1} X(k n-j), \quad k \in \mathbb{Z}, n \in \mathbb{N} .
$$

The resultant process has finite dimensional distributions similar to $X(k)$. Specifically for each $n$ we have that

$$
X^{(n)}(k)={ }_{d} n^{H} X(k), \quad n \in \mathbb{N} .
$$

The stationary process $X(k)$ that fulfills $(3)$ is called a self-similar stationary process, $H$-SSS, with Hurst exponent $H$. A typical example is the fractional Gaussian noise ( $\mathrm{fGn}$ ) given by the expression $X(k)=B^{(H)}(k+1)-B^{(H)}(k)$, which is the only known $H$-SSS Gaussian process [5].

There are several ways of studying the statistical properties of $X^{(n)}(k)$. The cumulants of the aggregate series, which are defined in terms of Taylor coefficients of the cumulant generating function, are considered in [5]

$$
g(t)=\log \mathrm{E}\left(e^{t X(k)}\right)=\sum_{m=1}^{\infty} t^{m} m !^{-1} \operatorname{cum}_{m} X(k),
$$

where $\operatorname{cum}_{m} X(k)=g^{(m)}(0)$. In [7] it is shown that the $m$ th order cumulants of an $\mathrm{H}$-SSS aggregate process scales according to a power law given by

$$
\operatorname{cum}_{m} X^{(n)}(k)=n^{m H_{s}} \operatorname{cum}_{m} X(k) .
$$

If a $H$-SSS process fulfills (5) $\forall n, m \in \mathbb{N}$, then $\left|\log \operatorname{cum}_{m} X^{(n)}(k)\right|$ behaves in such a way that its values scale linearly with those of $\log n$, with $m H_{S}$ coefficients that are a linear function of $m$. In other words, $m H_{s}=m H(m)$, i.e.

$$
\operatorname{cum}_{m} X^{(n)}(k)=n^{m H(m)} \operatorname{cum}_{m} X(k)
$$

In [5] it is shown that a generalization of a selfsimilar process to a multifractal process is given as follows: a stationary process $X(k)$ with $k \in \mathbb{Z}$ is a multifractal process if

$$
\log \left|\operatorname{cum}_{m} X^{(n)}(k)\right|=m H(m) \log n+c(m),
$$

for every $m, n \in \mathbb{N}$, allowing the exponent $H$ to vary with the order $m$.
The general form of $m H(m)$ is given by

$$
m H(m)=\alpha m+\beta,
$$

which corresponds to the linear fractal model, where the coefficients $\alpha$ and $\beta$ are determined directly during the fitting of the cumulants. In $[8,9]$ it is shown that the only known process of this kind takes the form

$$
m H(m)=m+2\left(H_{U}-1\right)
$$

and it is called a unifractal process.

In [5] the unifractal model is compared with the self-similar model using empirical flows. The analysis of Figures 3 and 4 in [5] puts in evidence that both models are capable of capturing the main trends of the real flows in the estimation of $H$, but also that none of them harmonizes with the locality phenomenon of the Hurst exponent.

Figure 1 gives an illustration of the locality phenomenon. In the current case the $X$-axis stands for $\log n$ and the $Y$-axis stands for log cumulant. The slope of the fitting curve crossovers from a small value to a notably larger value. Therefore the curve consists of three parts: a line segment with a gentler slope when log $n$ is small, the intermediate transition part, and another line segment with a steeper slope when log $n$ is large.

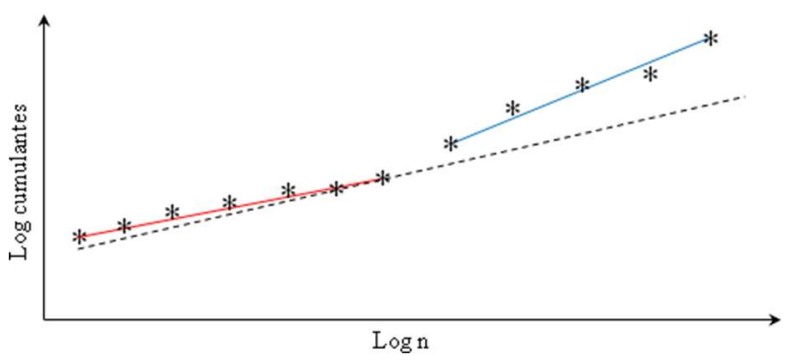

Figure 1. Illustration of the locality phenomenon.

\section{A Multifractal Model for the Locality Phenomenon}

Reference [10] reports that the PCA (Principal Components Analysis) of the spectrum of proper values that result from mixing fractional Brownian motion signals with different Hurst exponents $(H)$ 
produces a bi-scalar behavior. This precise fact is what motivates the statement of a multifractal flow traffic model capable of reproducing the locality phenomenon of the Hurst exponent.

Let us assume a network traffic process, $W(k)$, composed of the addition of two independent selfsimilar processes; $X_{1}(k)$ and $X_{2}(k)$, with Hurst exponents $H_{1}$ and $H_{2}$, respectively, i.e.

$$
W(k)=\alpha_{1} X_{1}(k)+\alpha_{2} X_{2}(k),
$$

where $\operatorname{Var}\left\{X_{1}\right\}=\operatorname{Var}\left\{X_{2}\right\}$ and the scale coefficients $\alpha_{1}$ and $\alpha_{2}\left(\alpha_{1}, \alpha_{2}>0\right)$ controlling the variance of the components of (10). Also, without loss of generality, that $H_{1}<H_{2}$. The same as for the case of (2), we can define the aggregate process $Z^{(n)}(k)$ through multiplexing increments $Z(k)$ given by the expression $Z(k)=W(k+1)-W(k)$, considering non-overlapping blocks of size $n$ as

$$
Z^{(n)}(k)=\sum_{j=0}^{n-1} X(k n-j), \quad k \in \mathbb{Z}, n \in \mathbb{N} .
$$

From independence, for the self-similar and unifractal models it is verified that

$$
\begin{aligned}
& \left|\operatorname{cum}_{m} Z^{(n)}(k)\right|= \\
& \left|\operatorname{cum}_{m}\left(\alpha_{1} X_{1}^{(n)}(k)+\alpha_{2} X_{2}^{(n)}(k)\right)\right|= \\
& C_{1}(m) n^{n H_{1}(m)}+C_{2}(m) n^{n H_{2}(m)}= \\
& C_{1}(m) n^{m+2\left(H_{1}(m)-1\right)}+c_{2}(m) n^{m+2\left(H_{2}(m)-1\right)}
\end{aligned}
$$

with $c_{1}(m)$ and $c_{2}(m)$ partially determined by the coefficients $\alpha_{1}$ and $\alpha_{2}$, respectively.

Then, if $c_{1}(m)>c_{2}(m)$, there is a unique positive solution $n^{*}$ (not necessarily integer) of the equation in terms of variable $n$

$$
C_{1}(m) n^{m+2\left(H_{1}(m)-1\right)}=C_{2}(m) n^{m+2\left(H_{2}(m)-1\right)} .
$$

Thus, it is easy to verify that

$$
\begin{aligned}
& \left|\operatorname{cum}_{m} Z^{(n)}(k)\right|= \\
& \left\{\begin{array}{l}
c_{1}(m) n^{m+2\left(H_{1}(m)-1\right)}, \text { para } n \ll n^{*} \\
C_{2}(m) n^{m+2\left(H_{2}(m)-1\right)}, \text { para } n \gg n^{*}
\end{array}\right.
\end{aligned}
$$

For every $m$ the logarithmic scale diagram of the $m$ th order cumulant of $Z^{(n)}(k)$ consists of three segments: a first linear segment with slope given for $m+2\left(H_{1}-1\right)$ when $n$ is small; an intermediate transition section (often very short), and finally a linear segment with slope $m+2\left(H_{2}-1\right)$ when $n$ is large. Therefore, the locality phenomenon of the Hurst exponent is put in evidence.

Figure 2 shows the locality phenomenon in the behavior of a $\mathrm{fGn}$ series with $H=0.6$ generated spectrally, while Figure 3 shows the phenomenon for a fGn series with $H=0.8$; Figure 4 shows the joint behavior of both series with $\alpha_{1}=\alpha_{2}$ in (10) [11].

The above shows in a simple manner that if a traffic process is formed from two independent self-similar additive components with different Hurst exponents, then the locality phenomenon is seen in the estimation of the $H$ exponent using cumulants. It is not difficult to see that this model can be extended to the case of more than two components with similar results.

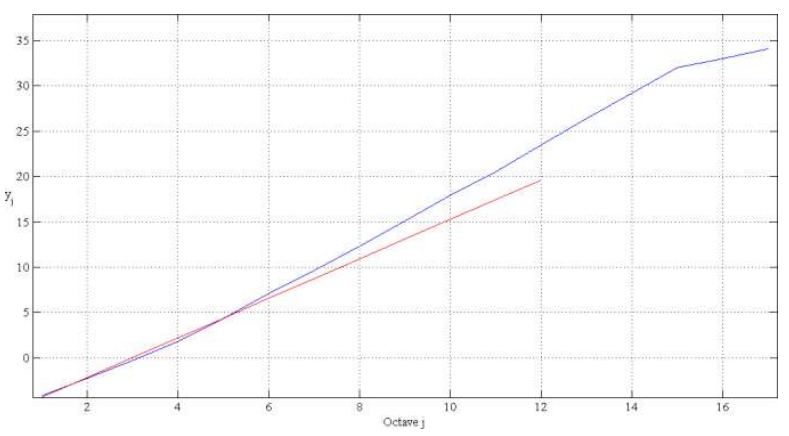

Figure 2. Phenomena in a $\mathrm{fGn}$ series with $H=0.6$.

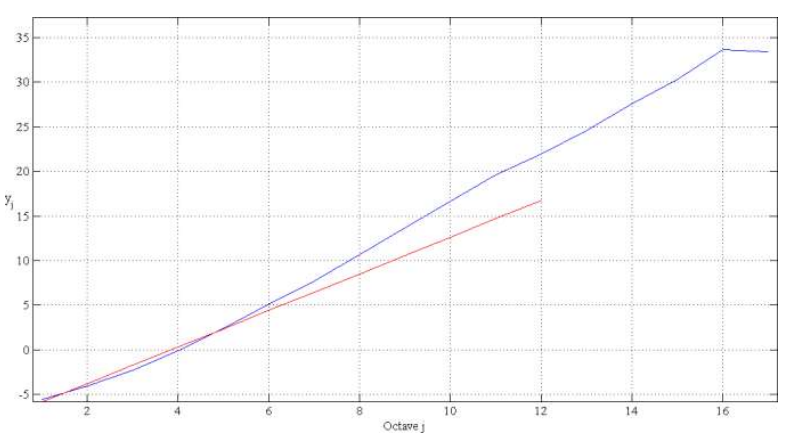

Figure 3. Phenomena in a $\mathrm{fGn}$ series with $H=0.8$. 


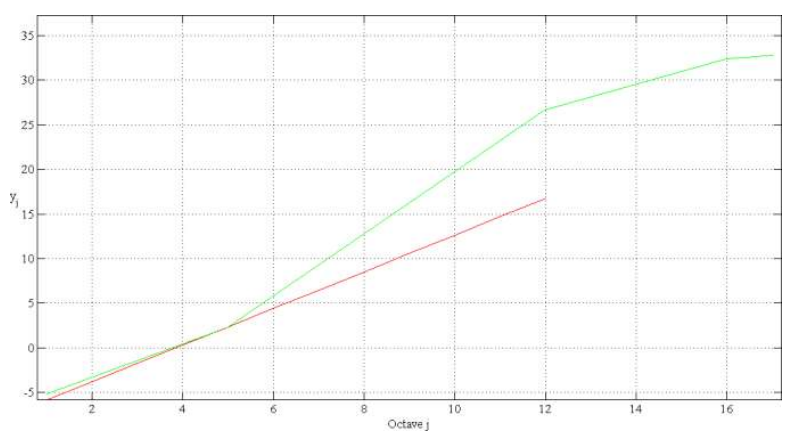

Figure 4. Phenomena observed in the joint behavior of the previous series with $\alpha_{1}=\alpha_{2}$ in (10).

\section{Conclusions}

It is interesting to notice that the theoretical inconsistency of a single value of the Hurst exponent and the locality phenomenon can be solved using a simple model that adds second order self-similar temporal series. On the other hand, it is possible to generate various processes with different Hurst exponents using any of the existing methods for the generation of second order self-similar series and then adding them. This agrees with the conclusion of [12], which states that it is necessary to multiplex various traffic sources to generate more appropriate simulations for the traffic flows.

Most of the previous models that deal with the origins of traffic flow self-similarity in high speed networks can be adopted, from the perspective of their application to the proposed model, with the purpose of stressing the coexistence in real flows of multiple self-similar heterogeneous components. It can therefore be stated that if in the On/Off model of [13] one assumes the existence of two different values for the Pareto exponents that control the distribution of the On periods and the Off periods of the sources, then the aggregate final process flow will present two different components in terms of their Hurst exponents and therefore the locality phenomenon will appear.

In general, for large coverage networks, i.e. with very complex structures, the data channel is shared by multiple sources in a manner that can be considered approximately independent and additive. Because of diversity of sources and transfer mechanisms, the incoming flow coming from each source has different Hurst exponents. This leads to the statement of the following conjecture: it is more probable to observe the locality phenomenon in traffic flows of trunk networks than of local area networks.

\section{References}

1. Millán, G., Kaschel, H. \& Lefranc, G. (2010). Discussion of the analysis of self-similar teletraffic with long-range dependence (LRD) at the network layer level. Int. J. Computers, Communications and Control, 5(5), 799-812.

2. Chen, Y.-D., Li, L., Zhang, Y. \& Hu, J.-M. (2009). Fluctuations and pseudo long range dependence in network flows: a non-stationary Poisson process model, Chinese Physics B, 18(4), 1373-1379.

3. Millán, G., Kaschel, H. \& Lefranc, G. (2010). A simple model for the generation of LRD self-similar traffic using piecewise affine chaotic onedimensional maps. Studies in Informatics and Control, 19(1), 67-78.

4. Millán, G. \& Lefranc, G. (2014). Application of the analysis of self-similar teletraffic with long-range dependence (LRD) at the network layer level, International Journal of Computer, Communications \& Control, 10(1), 62-69.

5. Terdik, G. \& Gyires, T. (2009). Lévy flights and fractal modeling of Internet traffic. IEEE/ACM Trans. Netw., 17(1), 120-129.

6. Sheluhin, O., Smolskiy, S. \& Osin, A. (2007). Self-Similar Processes in Telecommunications. Wiley: UK.

7. Millán, G., \& Lefranc, G. (2013). A fast multifractal model for self-similar traffic flows in high-speed computer networks Procedia Computer Science, 17, 420-425.

8. Iglói, E. \& Terdik, G. (2003). Superposition of diffusions with linear generator and its multifractal limit process, ESAIM Probab. Stat. 7, 23-88.

9. Molnár, S. \& Terdik, G. (2001). A general fractal model of Internet traffic. In Proc. 26th Annu. IEEE Conf. Local Computer Networks, p. 492-499.

10. Millán, G., Osorio-Comparán, R. \& Lefranc, G. (2021). Preliminaries on the accurate estimation of the Hurst exponent using time series. arXiv:2103.02091 [eess.SP].

11. Abry, P., Flandrin, P., Taqqu, M.S. \& Veitch, D. (2003). Self-similarity and long-range dependence through the wavelets lens. In: Doukhan, P., Oppenheim, G. \& Taqqu, M.S. (Eds.), Theory and Applications of Long-Range Dependence. Birkhäuser: Boston, MA, 527-556. 
12. Horn, G., Kvalbein, A., Blomskøld, J. \& Nilsen, E. (2007). An empirical comparison of generators for self-similar simulated traffic. Perform. Eval., 64(2), 162-190.

13. Millán, G. (2021). Traffic flows analysis in highspeed computer networks using time series. arXiv:2103.03984 [stat.AP]. 